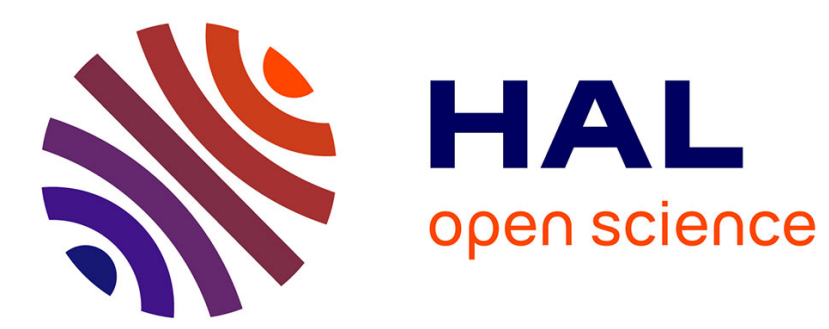

\title{
Le gutuater gaulois : idéologie et histoire
}

Christian Goudineau

\section{To cite this version:}

Christian Goudineau. Le gutuater gaulois: idéologie et histoire. Gallia - Archéologie de la France antique, 2003, 60, pp.383-387. 10.3406/galia.2003.3059 . hal-01909357

\section{HAL Id: hal-01909357 \\ https://hal.science/hal-01909357}

Submitted on 9 Jan 2020

HAL is a multi-disciplinary open access archive for the deposit and dissemination of scientific research documents, whether they are published or not. The documents may come from teaching and research institutions in France or abroad, or from public or private research centers.
L'archive ouverte pluridisciplinaire HAL, est destinée au dépôt et à la diffusion de documents scientifiques de niveau recherche, publiés ou non, émanant des établissements d'enseignement et de recherche français ou étrangers, des laboratoires publics ou privés.

\section{(이) $\$$}

Distributed under a Creative Commons Attribution - NonCommercial - NoDerivatives| 4.0 


\title{
LE GUTUATER GAULOIS
}

\section{Idéologie et histoire}

\author{
Christian Goudineau*
}

\begin{abstract}
Mots-clés. Gutuater, prêtre, guerre des Gaules, épigraphie, idéologie moderne, tradition manuscrite.
Résumé. L'auteur réagit à l'article de Yann Le Bohec publié dans un précédent volume de Gallia (58, 2001, p. 363-367). Contestant l'identification proposée entre le gutuater mentionné par quatre inscriptions gallo-romaines et le nom d'un Carnute cité par César et Hirtius, il rappelle que cette théorie, vieille d'un siècle, a été forgée pour démontrer que les druides avaient appelé à la révolte contre César, une thèse exploitée par des milieux marqués politiquement. C'est en connaissant cet arrière-plan que le lecteur peut examiner sérieusement la question.
\end{abstract}

Key-words. Gutuater, priest, Gallic war, epigraphy, modern ideology, written sources.

Abstract. The author gives his reaction to Yann Le Bohec's article published in a previous issue of Gallia (58, 2001, p. 363-367). He challenges the proposed identification of the gutuater mentioned in four Gallo-Roman inscriptions and the name of a Carnute man referred to by Caesar and Hirtius. He reminds the reader that the theory is a century old and was constructed to prove that the Druids had called for a revolt against Caesar, a thesis exploited by circles with a definite political bias. A knowledge of this background is essential if the reader is to seriously examine problem.

Schlagwörter. Gutuater, Priester, Gallischer Krieg, Epigraphik, Moderne Ideologie, Schriftliche Überlieferung.

Zusammenfassung. Der Verf. bezieht Stellung zu einem Aufsatz von Yann Le Bohec in einem der letzten Bände der Gallia (58, 2001, p. 363-367). Er stellt die darin vorgeschlagene Identifizierung des in vier gallorömischen Inschriften erwähnten gutuater mit dem bei Cäsar und Hirtius genannten Namen eines Carnuten in Frage und erinnert daran, daß die bereits ein Jahrhundert alte Theorie ursprünglich den Beweis dafür erbringen sollte, daß die Druiden zur Revolte gegen Cäsar aufgerufen hätten. Gewisse politische Kreise des 19. Jahrhunderts hatten sich diese These zunutze gemacht. Erst in Kenntnis dieses Hintergrundes kann der Leser den Sachverhalt gerecht beurteilen.

Übersetzt vom Stefan WIRTH

Le volume 58 de Gallia contient (p. 363-367) un article de Yann Le Bohec intitulé «Gutuater: nom propre ou nom commun? ". Ayant récemment travaillé sur l'historiographie s'attachant à la Gaule et aux Gaulois, j'ai souhaité ajouter quelques considérations à l'article de mon collègue, et je remercie Gallia de me l'avoir permis.

Le dossier se compose de deux séries de pièces, manuscrites et épigraphiques.

1. Au début du livre VII de la Guerre des Gaules, César écrit que la révolte (la grande insurrection, celle de 52) éclata chez les Carnutes, Cotuato et Conconnetodumno ducibus
(VII, 3). La tradition manuscrite, comme il est normal s'agissant de noms gaulois, n'est pas unanime. Il s'agit de duces, de " chefs », qui sont qualifiés par César de desperati homines, des insensés à la limite du suicidaire, qui massacrent peu après les citoyens romains établis à Orléans. Dans le livre VIII, écrit quelques années plus tard par Hirtius, celui-ci, relatant les événements de l'année 51, signale une expédition de César contre les Carnutes (VIII, 38). Le proconsul réclame la livraison du responsable de la révolte (de l'année précédente). Les manuscrits donnent au dit responsable le nom (à l'accusatif) Gutuatum, Gutruatum, Guttruatum, etc.

* Professeur au Collège de France, 11 place Marcelin-Berthelot, F-75005 Paris. 
2. Quatre inscriptions gallo-romaines (CIL, XIII, 1577, 2585,11225 et 11226 ) produisent le mot gutuater, dont Yann Le Bohec rappelle qu'il ne peut désigner qu'une charge religieuse. Trois de ces inscriptions ont été trouvées sur le territoire éduen, la quatrième chez les Vellaves.

Évidemment, dès la parution du Corpus inscriptionum latinarum, le rapprochement avait été suggéré - par Hirschfeld lui-même - entre les noms mentionnés par César et Hirtius (Cotuatus, Gutuatrus ou Gutuater, entre autres possibilités) et le gutuater des inscriptions. Il y a trois quarts de siècle, l'éditeur et traducteur du Bellum Gallicum aux Belles-Lettres, L.-A. Constans, résumait ainsi la question :

«César, VII, 3, 1, nomme comme instigateurs de la révolte des Carnutes Cotuatos et Conconnétodunnos (sic), desperati homines. S'agit-il ici [c'est-à-dire avec le Gutuatum du livre VIII] d'un troisième personnage, dont les deux autres n'auraient ćtć que les agents d'exécution ? Faut-il, au contraire, corriger au livre VII Cotuato en Gutuatro? ou bien écrire ici Cotualum? Les inscriptions nous apprennent que gutuater, nom commun, désigne une sorte de grand-prêtre : Cotuatus était-il gutuater, et Hirtius a-t-il pris la fonction pour le nom? Chacune de ces quatre hypothèses est défendable ${ }^{1}$."

Bien que prenant parti pour le nom propre, Constans demeure prudent : " chacune de ces quatre hypothèses est défendable ". L'écrit-il pour des raisons purement scientifiques ? Probablement. Mais, dans les années 1920, le choix entre telle ou telle solution est loin d'être innocent.

Impossible de comprendre si l'on ne se réfère à l'historiographie des $\mathrm{XIX}^{\mathrm{e}}$ et $\mathrm{XX}^{\mathrm{e}} \mathrm{s}$., aux interprétations des Gaulois, des Romains, de la guerre des Gaules, et surtout... des druides. Je résume en quelques mots.

Pendant longtemps, les druides ont été considérés comme des personnages plus ou moins éthérés, en tout cas extérieurs à l'histoire événementielle. Philosophes, moralistes, précurseurs de la religion chrétienne, ou, au contraire, faisant couler le sang de victimes humaines sur des autels barbares. Jusqu'au XIX ${ }^{\mathrm{e}}$ s., ces images furent totalement déconnectées de la "politique ", d'autant que l'on ne savait les rattacher à une époque précise. Les mouvements "druidiques " qui naquirent au XVIII"s. en Angleterre ne s'occupaient pas de la guerre des Gaules: Celtes et Gaulois représentaient l'origine de l'humanité dans l'Occident, les druides étaient les plus anciens prêtres.

Voilà que, au début du XIX ${ }^{e}$ s., avec Amédée Thierry ${ }^{2}$ puis Henri Martin ${ }^{3}$, naît une histoire de France dont

1. L.A. Constans, César, Guerre des Gaules, Paris, Les Belles Lettres, 1962, p. 309 (la première édition remonte à 1926).

2. A. Thicrry, Histoire des Gaulois depuis les temps les plus reculés jusqu'à l'entière soumission de la Gaule à la domination romaine, Paris, 1828.

3. H. Martin, Histoire de France depuis les temps les plus reculés jusqu'en 1789, t. I, Paris, 1837. l'origine ne se rattache plus à Clovis et aux Francs, mais qui remonte aux Gaulois, les " ancêtres". On relit les textes anciens avec un oil nouveau. Vont se développer la paléographie, en même temps que se créent les sociétés savantes qui auront tant d'importance pour l'histoire et l'archéologie. Je rappelle aussi d'un mot l'entreprise de Napoléon III, l'Histoire de Jules César, et notamment son deuxième volume consacré à la guerre des Gaules.

Ces livres d'histoire centrés sur le $\mathrm{I}^{\mathrm{er}} \mathrm{s}$. avant J.-C. eurent une conséquence inattendue : ils mirent en lumière l'action d'un chef éduen, Divitiac, allié de César, dont Cicéron (seul) avait révélé la qualité de druide. Nul ne s'indignait de cette complicité, car, si l'on plaignait l'armée de Vercingétorix et le chef lui-même d'avoir été défaits à Alésia, nul ne regrettait l'entrée de la Gaule dans le monde de la civilisation. La vaillance des soldats gaulois, le sacrifice héroïque de leur chef permettaient que cette entrée se fît "le front haut ».

Il en alla bien autrement après la défaite de 1870. Outre que la guerre avait été assimilée aux campagnes de 52 avant J.-C., son issue déclencha des comparaisons d'une violence inouie : la France avait été trahie par les dignitaires de l'Empire, les « colliers d'or ", notables et ecclésiastiques, de même que Vercingétorix avait perdu en raison de la traîtrise des nobles Éduens et des druides, qui souhaitaient la victoire de César. Dans l'un et l'autre cas, les victimes, c'était la patrie, c'était le peuple. Telle était la thèse des " républicains ", que ne pouvaient guère contester leurs adversaires ${ }^{4}$.

Divine surprise ! Surgissent, à la fin du $\mathrm{XIX}^{\mathrm{c}}$ et au début du $\mathrm{XX}^{\mathrm{e}}$ s., les volumes du CIL, XIII. Les mentions d'une charge religieuse de gutuater (quoique gallo-romaine) sont rapprochées des passages de Hirtius et de César : c'est un gutuater qui fut mis à mort, c'est donc lui qui avait déclenché l'insurrection. Coluato doit donc se lire Gutruato ou Gutruatro, et il faut traduire non par un nom propre mais par « le gutuater » - autrement dit, " le prêtre » ou " le druide ». Telle est la nouvelle théorie opposée à la " thèse républicaine ". Les polémiques furent d'une violence qu'on n'imagine plus aujourd'hui parce que chargées de considérations historiques qui se transformaient en connotations idéologiques et en pétitions politiques (autour de la laïcité et de l'anticléricalisme). Lorsque Yann Le Bohec (qui cite deux articles de 1977 et 1987) conclut que la proposition (gutuater, nom commun) " apporte un argument aux historiens qui

4. Par exemple E. Bosc et L. Bonnemère, Histoire nationale des Gaulois sous Vercingétorix, Paris, 1882. Deux extraits, p. 209: "Nous avons à plusieurs reprises signalé le rôle odieux des druides. Nous avons dit que Divitiac guerroyait commc un simple collier d'or "; p. 448: " [César] voulait avoir l'air de respecter la religion; les druides avaient assez fait pour lui ". Voir A. Simon, Vercingétorix el l"idéologie française, Paris, 1989, notamment p. $71,85,95$ sq. 
accordent une importance particulière au clergé celtique dans la révolte de 52 avant J.-C. ", sa formule est si calme, si neutre (en dépit de l'expression "clergé celtique ») que quiconque ignore l'utilisation de l'argument il y a quelques décennies ne saurait comprendre qu'il fut largement exploité pour propager des thèses proches de l'extrême droite d'alors.

Pour prendre la mesure du phénomène, un simple exemple, tiré d'un roman paru en 1937. Des vingt ou trente pages décrivant une réunion dans le lieu druidique consacré, au cœur du pays carnute, je ne cite que quelques lignes :

«Aujourd'hui le pays des Ancêtres est en danger. Dieux le veulent! Dieux le veulent! guerre contre les Romains !

"Alors, les Bardes s'effaçant, procède le Druide des Druides, le Vénérable indépendant de toutes autorités, Chef Suprême élu à vie en dehors et au-dessus des peuples, le "Roi des Sacrifices" de cette Gaule sanctuaire de la religion du Sacrifice.

"Il dit, écouté avec l'aveugle obéissance que la nation entière - état d'Église - voue au Sacerdoce : "Vous savez tous quelle servitude attend le pays insurgé et les chefs : sur le Pays, après le pillage, s'abattront l'impôt, les exactions, l'oppression ; sur les chefs, la mort, le supplice ou la prison perpétuelle; sur les combattants, l'esclavage. IL. FAUT FAIRE LA GULRRE DÉSESPÉRÉE ! [... " "5."

Des textes comme celui-là, il est aisé d'en produire des dizaines. La démonstration est toujours la même : c'est dans la forêt sacrée des Carnutes, sous les auspices voire à l'instigation des druides, que la Gaule a décidé de se lever contre César. La religion est indissociablement liée à la patrie. Le gutuater fait son ouvre.

Dans un tel contexte, les historiens ne se sentent pas forcément à leur aise. Alors que ni Amédée Thierry, ni Henri Martin, ni Napoléon III n'avaient mis en doute que les noms cités par César et Hirtius ne fussent des noms propres, la question se posa à Camille Jullian. Dans son Vercingétorix, paru au début 1901, il écrit :

"Le jour fixé, deux chefs carnutes, Gutuatr et Conconnetodumn, hommes d'audace et d'aventure, les "risque-tout" de l'indépendance, pour parler comme César, donnent le signal, réunissent leurs hommes et entrent dans Génabum. Ils vont droit aux maisons où habitaient les citoyens romains, les égorgent sans trouver de résistance et font main basse sur leurs biens. Cita, le chef de l'intendance de César, périt comme les autres ${ }^{6}$."

5. M. L.cblond, A. Leblond, Vie de Vercingétorix, la jeunesse, la victoire, Paris, 1937, p. 29.

6. C. Jullian, Vercingétorix, Paris, 1901, p. 119.
Plus loin, relatant les événements de l'an 51 :

"La marche de César, depuis la Meuse jusqu'à la Dordogne, marqua la Gaule d'une traînée sanglante. Chez les Carnutes, visités une troisième fois de l'année par les armées romaines, il put enfin, après une étonnante chasse à l'homme, mettre la main sur Gutuatr. Lui et ses légions avaient à tout prix besoin, pour être en règle avec les dieux de Rome, du corps de l'homme qui avait donné le signal de la lutte à toute la Gaule et à Vercingétorix lui-même. L'exécution du chef carnute fut faite en vue de toute l'armée, et il semble que César ait permis à chaque soldat de prendre un peu du sang de celui qui avait versé le premier sang romain. Il fut battu de verges par tous ceux qui se présentèrent, et il n'était guère plus qu'un cadavre quand on se décida à le frapper de la hache ${ }^{7}$."

Jullian prend donc parti pour la solution traditionnelle : il s'agit de noms propres, Coluatus et Gutuater représentent le même nom, qu'il se décide à orthographier Gutuatr.

Quelques années plus tard, en 1907, dans le tome II de sa grande Histoire de la Gaule, le texte courant confirme cette position :

"Au jour fixé, les Carnutes donnèrent le signal. Deux hardis compagnons, Gutuatr et Conconnetodumn s'étaient décidés à tous les risques pour la cause de la liberté. »

De même, un an et demi plus tard, c'est Gutuatr que César réclame aux Carnutes ${ }^{8}$.

Cependant, une note commente le premier passage, et elle dit ceci :

"Les mss. ont (VII, 3,1) Cotuato et Conconnetodumno; en revanche (VIII, 38, 3), Gutruatrum, Gutuatrum, Guttruatum, Guttruatrum. Je crois bien que l'orthographe était Gutuater, et il serait possible que ce fût un titre de prêtrise. "

"Il serait possible »: l'enthousiasme n'est pas délirant, ce que confirme la relégation en note.

Sautons un demi-siècle. Michel Rambaud - qui, pourtant, insiste sur la «force d'opposition » que représentent les druides et Jacques Harmand - qui ne partage guère les thèses du premier - inclinent à penser que CotuatusGutuater représente un nom propre, même si tous deux jugent le personnage «mystérieux » ou " énigmatique " 9 . Les spécialistes de l'histoire des religions, quoique tentés

7. C. Jullian, Vercingétorix, Paris, 1901, p. 338.

8. C. Jullian, Histoire de la Gaule, Paris, 1907, p. 580 et p. 629 de la réédition de 1993.

9. M. Rambaud, L'art de la déformation historique dans les Commentaires de César, Paris, 1966, p. 324. J. Harmand, Vercingétorix, Paris, 1984, p. 135. 356 et 367. 
par l'idée que César et Hirtius aient mentionné des druides, restent généralement prudents ${ }^{10}$, sauf parfois ceux qui traitent directement le sujet " druides " ${ }^{11}$.

Dans la mesure où, aujourd'hui, nous sommes libërës (je l'espère) des querelles d'autrefois, où les Gaulois ne jouent plus un grand rôle dans les débats politiques, où l'on n'en est plus à invoquer les druides pour pourfendre les " anticléricaux ", il est sans doute possible de reprendre calmement le dossier. Je présenterai donc les observations suivantes.

1. Évidemment, il est incontestable que, dans les inscriptions, gutuater représente une fonction religieuse. Laquelle? Nous l'ignorons. Quelle fut sa diffusion? Faible, apparemment.

2. Le gutuater des inscriptions peut-il être identifié aux noms cités par Hirtius el César? Je ne le crois pas, pour plusieurs raisons. D'abord, parce que César et Hirtius écrivent un ouvrage destiné à des lecteurs romains - pour l'essentiel, des sénateurs et chevaliers de Rome. Lorsque l'on s'adresse à des compatriotes n'ayant aucune idée des institutions ou des mours des pays étrangers dont on leur parle, il n'est que deux solutions - celles que tous les " explorateurs " ou conquérants ont mises à l'ceuvre. Soit on transpose, soit on explique. César a transposé souvent - notamment dans le fameux passage où il nomme les dieux de la Gaule - mais il a aussi fait ouvre pédagogique, plus d'une fois. Par exemple, lorsqu'il explique à ses lecteurs ce qu'est un vergobrct : "c'cst lc magistrat suprême, il est nommé pour un an, et a droit de vie et de mort sur ses concitoyens " (I, 16), explication qui sera reprise plus tard (VII, 32). Hirtius, lui aussi, se livre à de la pédagogie, parfois pesante, rappelant où se trouve tel ou tel peuple, les événements précédents, les actions de César. Eût-il laissé passer un terme comme gutuater sans dire qu'il s'agissait d'une charge

\footnotetext{
10. J. Vendryes, "L a religion des Celtes", in Les religions des Celtes, des Germains et des anciens Slaves, Mana, t. III, 1948, p. 304-305. J. de Vries, La religion des Celles, Paris, 1977, p. 222-223 (« il se pourrait que le mot gutuatros [chc\% César] ait été presque synonyme de druide "). En revanche, J.-L. Brunaux, Les religions gauloises, Paris, 1996, p. 24, écrit à propos d' " un nom de prêtre gaulois, le gutuater ": «[Ce dernier] est le nom d'un Carnute qui est accusé d'avoir fomenté la révolte contre les Romains, mot que l'on retrouve sur plusieurs inscriptions galloromaines et qui paraît désigner un type de prêtre ".

11. Voir par exemple, F. Le Roux, C.-J. Guyonvarc'h, Les druides, 1986, p. 444. Voir aussi l'entrée Gutrualos dans V. Kruta, Les Celles, histoire et dictionnaire, Paris, 2000, p. 654 : "Fonctionnaire religieux de haut rang [...] connu par les inscriptions de Gaule d'époque romaine et par César (où le titrc est confondu avec un nom de personne) ". La thèse que César ou Hirtius ont " confondu », thèse souvent mise en avant par les partisans du nom commun, parait difficile à soutenir, dans la mesure où le proconsul est entouré de Gaulois de haut rang (y compris, peutêtre, de druides, comme on voit par l'exemple de Diviciac) et notamment d'Éduens, sur le territoire desquels ont été trouvées trois des quatre inscriptions gallo-romaines!
}

religieuse ? C'est invraisemblable. Et pourquoi n'eût-il pas cité son titulaire ? Car quiconque lit du début à la fin le De Bello Gallico se rend compte que tous les intervenants sont nommés. Aucun n'est seulement signalé par la charge qu'il occupe. Hirtius écrivit le livre VIII après la mort de César (15 mars 44), lui-même mourut en avril 43. Comment aurait-il désigné le responsable (selon lui) de la révolte des Carnutes - huit ans après les événements - par ce titre incompréhensible de gutuater?

Un argument d'ordre stylistique va dans le même sens. Dans ce livre VIII, Hirtius commente la mort du chef bellovaque Correus, lequel vient d'être tué les armes à la main (VIII, 21). Des députés sont envoyés à César. Non sans hypocrisie, ils se félicitent de la mort de Correus qui, à les croire, avait dépossédé leur Sénat au profit de la "plèbe ignorante ». Lisons : Correus, auctor belli, concitator multiludinis, esset interfectus.

Reportons-nous au passage dans lequel le même Hirtius narre le supplice du Carnute Gutuater (vel sim.) : principem sceleris illius et concitatorem belli, Gutuatrum, ad supplicium deposcit. Le parallélisme n'est-il pas frappant? Emploi des mots concitator, bellum, construction analogue. Pourquoi, dans le premier cas, Hirtius citerait-il un nom propre et, dans le second, un nom commun?

Reste l'éventuelle coïncidence entre un nom propre et un nom commun. Mais serait-elle si troublante? Dans toutes les langues, on en relève nombre d'exemples: combien de Lévêque ou Leprêtre aujourd'hui en France? "Vercingétorix " (" grand roi des guerriers ") n'est-il pas, à l'origine, un nom de fonction, au point que Michelet (écrivant avant la découverte des monnaies à la légende du chef arverne) disait "le vercingétorix " comme d'autres suggèrent de lire "le gutuater " chez César et Hirtius ?

3. Subsiste un point, en l'occurrence, secondaire. Si le Gutuater ou le Gutuatus de Hirtius est le nom d'un chef carnute, peut-on l'identifier à celui du Cotuatus (à l'ablatif Cotuato) signalé par César?

Rappelons les hésitations des manuscrits, dues en partie au fait que, pour unc orcillc romaine, certains sons celtiques étaient difficiles à transcrire en latin. Le nom du second chef, celui qui s'est joint en 52 à Cotuatus, Conconnétodumnus, connaît aussi des variantes dans la tradition manuscrite. Or, nous avons la chance de le retrouver dans l'épigraphie de Mediolanum Santonum (Saintes), où il est écrit CONGONNETODVBNVS ${ }^{12}$. On voit la difficulté de noter l'occlusive, tantôt $\mathrm{C}$, tantôt G; le nom d'Orléans, également, est écrit tantôt Cenabum, tantôt Genabum. Il en allait ainsi pour les voyelles $\mathrm{O}$ et $\mathrm{U}$. La finale de Correus ou de Commius se prononce-t-elle -us ou -os? Le nom de

12. Voir L. Maurin, Inscriptions latines d'Aquitaine, Santons, Bordeaux, 1994, p. 96 et 124 sq. à propos de CIL, XIII, 1040, 1042-1045. 
Dumnacos, cité en VIII, 26 sq., est orthographié, selon les manuscrits, Dumnacos, Domnacus, Deunacos ${ }^{13}$.

Donc, comment ne pas conserver la théorie ancienne, à savoir la concordance entre COTVATVS et GVTVATVS (ou équivalents ; à décliner, évidemment) ${ }^{14}$ ?

13. Voir P.-Y. Lambert, La langue gauloise, Paris, 1994, p. 41-46.

14. Le fait que César n'ait pas demandé la livraison de Conconnétodumnos peut s'expliquer simplement par la mort de ce personnage : les occasions n'avaient manqué ni en 52 ni en 51. L'acharnement du proconsul à punir le Carnute suppose de nombreuses conversations roulant autour de celui-ci. On croira malaisément qu'un quiproquo ait pu régner aussi longtemps.
Pour conclure. De la coincidence (peut-être approximative) entre un nom propre (dont on ignore la graphie exacte) du I ${ }^{\mathrm{er}}$ s. avant J.-C. et celui d'une charge religieuse celtique (attestée ailleurs et plus tard), a été conçue, essentiellement dans les milieux "anti-républicains » (pour reprendre les catégories de l'époque), souvent d'extrême droite, la théorie selon laquelle le " clergé druidique " avait appelé la Gaule à la révolte contre César. Elle me paraît scientifiquement inacceptable. Aux lecteurs d'en juger. Mais on ne saurait le faire en ignorant les arrièreplans historiographiques et idéologiques qui entourent le gutuater. 